\title{
Terapia manual no tratamento da espondilólise e espondilolistese: revisão de literatura
}

\section{Manual therapy for treating spondylolysis and spondylolisthesis: a literature review}

\author{
Fabrício José Jassi', Leila Suzuki Saita², Ana Carolina Pimenta Grecco², Margarete Kazue Tamashiro², Danilo Santos \\ Catelli ${ }^{3}$, Paulo Roberto Carvalho do Nascimento', Heloyse Uliam Kuriki', Rubén de Faria Negrão Filho
}

Estudo desenvolvido no LAFAMH - Laboratório de Fisioterapia Aplicada ao Movimento Humano da Unesp - Universidade Estadual Paulista Júlio de Mesquista Filho, campus de Presidente Prudente, SP, Brasil

1 Fisioterapeutas; mestrandos em Fisioterapia na Unesp Presidente Prudente

2 Fisioterapeutas especializandas em Terapia Manual e Técnicas Osteopáticas na Universidade Estadual do Norte do Paraná, campus de Jacarezinho, PR

3 Educador físico; mestrando em Bioengenharia na USP São Carlos - Universidade de São Paulo, campus de São Carlos, SP

4 Fisioterapeuta; doutoranda em Bioengenharia na USP São Carlos

5 Prof. Dr. do Depto. de Fisioterapia da Unesp Presidente Prudente

ENDEREÇO PARA CORRESPONDENCIA:

Fabrício J. Jassi R. Palmira Ferreira Santos 63 Santos Dumont 86430-000 Santo Antônio da Platina PR e-mail: jassi_jose@hotmail.com O estudo recebeu apoio da Fapesp - Fundação de Amparo à Pesquisa do Estado de São Paulo

APRESENTAÇÃO out. 2009

\section{ACEITO PARA PUBLICAÇÃO} ago. 2010
Resumo: A espondilólise é um defeito na pars interarticularis da vértebra com descontinuidade óssea do segmento intervertebral; a progressão do defeito resulta em deslizamento de uma vértebra sobre a outra, chamado espondilolistese, o que pode provocar dor. O tratamento não-cirúrgico é a escolha inicial na maioria dos casos de espondilolistese, mas poucos estudos verificam a eficácia dos tratamentos conservadores. O objetivo deste estudo foi realizar uma revisão da literatura sobre esses tratamentos, sobretudo no que concerne à terapia manual, a fim de ajudar os terapeutas na prescrição de intervenções eficazes. Os resultados mostram que tanto a terapia manual como a fisioterapia convencional apresentam efeitos benéficos na redução da dor lombar e na melhora funcional do paciente. As terapias manuais envolvem manipulação da coluna vertebral e articulação sacroilíaca, músculo-energia e alongamento dos músculos afetados. Exercícios de estabilização lombopélvica, fortalecimento dos músculos posturais e alongamento dos isquiotibiais e psoas também foram considerados importantes. O paciente deve ser avaliado individualmente em seu quadro clínico e radiográfico para determinação do plano de tratamento. Dentre as opções conservadoras de tratamento encontradas, nenhuma se mostrou conclusivamente superior às outras e todas podem ser incluídas no tratamento sintomático de pacientes com espondilólise/listese.

Descritores: Espondilólise; Espondilolistese/reabilitação; Manipulações musculosqueléticas; Modalidades de fisioterapia

Abstract: Spondylolysis is a defect in the pars interarticularis of a vertebra with a disruption in the intervertebral segment. Progression of the defect leads to one vertebra slipping over another - which is called spondylolisthesis - which may cause lowback pain. Non-surgical treatment is the initial course of action in most cases of spondylolisthesis. However, few studies have assessed the efficacy of conservative treatment. The purpose of the present study is to review literature on conservative treatment for spondylolysis/listhesis, especially manual therapy, in order to guide practitioners for effective intervention. Results show that both manual therapy and conventional physiotherapy were effective in relieving low-back pain and beneficial for patient's functional outcome. Manual therapy involved spine manipulation, sacroiliac joint manipulation, muscle-energy techniques and stretching affected muscles. Stabilizing lombopelvic exercises, postural muscles strengthening, and hamstring and psoas stretching were also considered important in treating spondylolysis/listhesis. Each case's clinical and radiological features must be individually considered in order to determinate therapeutic strategy. Among non-surgical options, none has proved better than others and all may be included in symptomatic treatment of patients with spondylolysis/listhesis.

Key words: Musculoskeletal manipulations; Physical therapy modalities; Spondylolysis; Spondylolisthesis/rehabilitation 
INTRODUÇÃO

Espondilólise e espondilolistese são duas condições que envolvem mudanças diretamente na vértebra. A espondilólise é definida como um defeito com descontinuidade óssea do segmento intervertebral, região da lâmina entre os processos articulares superiores e inferiores. A progressão do defeito pode resultar em espondilolistese, que é a subluxação de duas vértebras adjacentes, evidente no raio-x de projeção lateral e oblíqua como uma linha de separação posterior ao corpo da vértebra1,2. Estudos indicam que 50 a $81 \%$ dos casos de espondilólise estão associados com espondilolistese ${ }^{3}$.

A ocorrência da espondilolistese é maior na coluna lombar, principalmente entre os níveis L5-S1, predispondo à lombalgia, sobretudo em crianças e adolescentes 4,5. Estudos epidemiológicos mostraram que a incidência de espondilólise está relacionada com a idade, herança genética, gênero, raça e nível de atividade $^{2}$, manifestando-se freqüentemente durante a fase de crescimento, dos 8 aos 20 anos. O risco declina na meia idade e aumenta ligeiramente entre os 60 a 80 anos $^{6}$. Além desses fatores, atividades que requerem hiperextensão ou hiperflexão da coluna lombar aumentam o risco de espondilólise/listese. Esportes como ginástica, futebol, mergulho, luta, levantamento de peso e remo apresentam incidência excepcionalmente alta dessas patologias 2,7. Embora essa condição muitas vezes se desenvolva cedo na adolescência, normalmente não é detectada até a idade adulta. Crianças e adolescentes geralmente suportam a dor lombar por muitos anos antes de serem avaliadas por um médico 8 .

A espondilolistese é classificada em cinco categorias baseada na sua etiologia: displástica, ístmica, degenerativa, traumática e patológica9 (Quadro 1).

Geralmente a espondilolistese é assintomática 3 , porém dor lombar e ciática bilateral até as nádegas ou face posterior das coxas podem se manifestar ${ }^{10}$. A dor é leve ou moderada, inicialmente obtusa, que se exacerba com movimentos de extensão e rotação 2,5 . No exame físico, a dor pode ser reproduzida no teste de extensão lombar com apoio unipodal no qual o paciente realiza descarga de peso em apenas um membro inferior enquanto estende a coluna 11 . Verifica-se à palpação um degrau entre os processos espinhosos das vértebras lombares acometidas em caso de espondilolistese ${ }^{5}$.

Apesar da natureza degenerativa da doença, a amplitude de movimento pode ser normal e ocasionalmente aumentada 5 . Alguns pacientes podem ser capazes de tocar seus dedos dos pés na flexão anterior do tronco como resultado da hipermobilidade segmentar ou do relaxamento ligamentar generalizado 5 . Entretanto, em casos severos, uma limitação da amplitude de movimento de flexão do tronco pode ser observada 5 . A progressão da patologia resulta em tensão dos isquiotibiais, retroversão da pelve, fraqueza dos músculos abdominais e postura flexionada dos quadris e joelhos. A marcha pode estar alterada com os membros inferiores rígidos, passos curtos e rotação pélvica 5 . Sintomas neurológicos acompanham os graus mais severos de espondilolistese, normalmente do tipo displástico, no qual as raízes do nervo lombosssacro podem ser comprimidas atrás da face póstero-superior do sacro. Além disso, algum grau de dor radicular não é incomum na espondilolistese ístmica de longa data. Não obstante, sinais neurológicos objetivos são excepcionais 4 .

Para confirmar o diagnóstico de espondilolistese a lesão da pars articularis

Quadro 1 Classificação da espondilolistese 2,4

\begin{tabular}{|c|c|c|}
\hline & $\begin{array}{l}\text { Displástica } \\
\text { (Congênita) }\end{array}$ & $\begin{array}{l}\text { Anormalidade na formação do arco neural no nível L5-S1 } \\
\text { que permite um deslizamento de L5 sobre S1. Os sintomas } \\
\text { podem se desenvolver após } 8 \text { anos de idade. }\end{array}$ \\
\hline II & $\begin{array}{l}\text { Ístmica } \\
\text { (Espondilolítica) }\end{array}$ & $\begin{array}{l}\text { A mais comum, causada pela falência por fadiga dos pares } \\
\text { articulares devido a estresse repetitivo em extensão/torção. } \\
\text { Sua incidência é maior no nível L5-S1 de adolescentes e } \\
\text { adultos jovens. Uma cifose toracolombar é uma das causas } \\
\text { predisponentes. }\end{array}$ \\
\hline III & Degenerativa & $\begin{array}{l}\text { Degeneração do disco e/ou instabilidade intersegmentar. } \\
\text { Uma hipomobilidade em L5-S1 pode causar hipermobi- } \\
\text { lidade em L4-L5, tornando prováveis alterações } \\
\text { degenerativas como deslizamento anterior. Ocorre mais } \\
\text { freqüentemente em mulheres acima dos } 40 \text { anos. }\end{array}$ \\
\hline IV & Traumática & $\begin{array}{l}\text { São as fraturas agudas dos pares articulares e curam-se bem } \\
\text { com imobilização. }\end{array}$ \\
\hline V & Patológica & $\begin{array}{l}\text { Metástases e doença reumática são suas causas mais } \\
\text { comuns. Outras patologias como tuberculose, doença de } \\
\text { Paget, doença de Albers-Schönberg, artrogripose e sífilis } \\
\text { podem enfraquecer o tecido da vértebra e torná-la mais } \\
\text { suscetível a danos. Assim como acontece na traumática, } \\
\text { esses casos freqüentemente acometem todo o segmento } \\
\text { vertebral, não sendo um problema particular dos pares } \\
\text { articulares. }\end{array}$ \\
\hline
\end{tabular}

deve ser identificada em imagens radiográficas 12 . Espondilólise nos estágios iniciais pode não ser visível nos planos radiográficos. O exame de cintilografia óssea irá revelar um aumento da compressão na pars interarticularis indicando reação de estresse ${ }^{2}$. Os parâmetros mais importantes para determinar a progressão da patologia são a quantidade de escorregamento e o ângulo entre as vértebras supra e subjacentes 4 .

O tratamento não-cirúrgico é a escolha inicial na maioria dos casos de espondilolistese, com ou sem sintomas neurológicos, além de ser a principal forma de tratamento das dores lombares. Não há, entretanto, estudos prospectivos randomizados que estabeleçam um protocolo ideal de tratamento ${ }^{5}$. Na maioria dos casos sintomáticos de espondilólise e espondilolistese o tratamento conservador é recomendado para reduzir a dor, restaurar a amplitude de movimento e a função, e para fortalecer e estabilizar os músculos espinhais 13 .

Existe uma lacuna óbvia no conhecimento concernente à efetividade dos tratamentos conservadores, que podem incluir modalidades para alívio da dor, como eletro e termoterapia, exercícios de fortalecimento dos músculos posturais, limitação e reeducação para as atividades diárias, imobilização com colete lombar, 
terapia manipulativa, massagem, tração, entre outros ${ }^{14}$.

O objetivo do presente estudo foi realizar uma revisão da literatura sobre os tratamentos conservadores da espondilólise/listese, sobretudo no que envolve a terapia manual, a fim de ajudar os terapeutas na escolha de intervenções eficazes para seus pacientes e fornecer subsídios para futuras pesquisas.

\section{METODOLOGIA}

Foi realizada uma busca de publicações referentes ao tratamento conservador da espondilólise e espondilolistese, com enfoque na terapia manual, osteopatia e quiropraxia, publicadas em inglês entre 1986 e 2008. A pesquisa em três das principais bases de dados - Pubmed, Science Direct e Web of Science - permitiu a seleção dos estudos apropriados, mediante os descritores spondylolisthesis, spondylolysis, chiropractic, ostheopatic, manipulation, exercises e treatment, de forma individual e combinada.

Dentre os estudos encontrados foram selecionados aqueles onde a estrutura envolvida era a coluna lombar, englobando a faixa dos 10 aos 57 anos, evidência radiográfica da patologia e tratamento conservador. Foram excluídos estudos nos quais os sujeitos apresentavam deficit neurológico ou autonômico, fratura, anormalidade óssea ou problema na coluna vertebral não-relacionado à espondilólise/ listese. Os critérios de exclusão foram determinados para limitar a influência de fatores externos sobre os resultados da intervenção e para limitar o estudo aos sintomas de espondilólise e espondilolistese. Além disso, os desfechos dos estudos deveriam incluir um ou mais dentre os seguintes: amplitude de movimento, dor, força muscular, avaliação da melhora funcional, grau de satisfação do paciente, qualidade de vida, eficácia da intervenção e/ou custo-benefício do tratamento. Ao final da seleção, um total de sete artigos 2,3,5,12,14,15,16 atenderam às especificações estabelecidas.

\section{RESULTADOS}

No que se refere à questão da eficácia das intervenções fisioterapêuticas no tratamento de dor lombar relacionada à espondilólise e à espondilolistese, $\mathrm{McNe}$ ely et al. 5 , em uma revisão sistemática, encontraram poucos estudos prospectivos. Dos 52 estudos por eles revisados, apenas 2 preencheram todos os critérios de seleção, os quais consideravam aspectos como confiabilidade, replicabilidade e relevância para a área. Esses autores verificaram que exercícios específicos para os músculos estabilizadores do tronco (abdominais profundos e multífido lombar) têm um efeito positivo sobre a dor lombar e a debilidade funcional relacionada a ambas as patologias, sendo mais eficazes do que tratamentos generalizados como nadar, caminhar, fazer ginástica e até mesmo outros exercícios de tronco inespecíficos. Também há evidências indicativas de que a combinação de exercícios em extensão, colete em extensão e instruções educativas são benéficos, embora não seja possível separar os efeitos individuais do programa combinado.

Contrariamente, Sinaki et al.15 encontraram que exercícios em flexão e fortalecimento isométrico são mais indicados do que exercícios apenas em extensão, verificando, após 3 meses, uma incidência de $27 \%$ de dor moderada a severa nos pacientes que realizaram exercícios somente em flexão, contra $67 \%$ naqueles que realizaram exercícios somente em extensão. Após 3 anos de acompanhamento, a dor moderada a severa caiu para 19\% no grupo de exercícios de flexão e permaneceu $67 \%$ no grupo de exercícios de extensão. Uma limitação desse estudo, entretanto, foi a falta de um grupo controle (nenhum exercício). Conseqüentemente, a eficácia real da flexão versus nenhum exercício não foi determinada.

Excoffon e Wallace16, ao estudar um paciente adulto masculino com herniação progressiva do disco lombar e espondilolistese ístmica de grau IV, utilizaram a abordagem quiropráxica no manejo e reabilitação do paciente. Um procedimento distrativo de Thompson drop, na posição prona, foi usado para acessar a região sacroilíaca e lombossacra da coluna. Esses ajustes foram executados em mesa Zenith Hi-Lo. Também foram realizados ajustes torácicos na posição prona, com técnica transversal dupla diversificada e ajustes cervicais executando procedimentos diversos. O paciente obteve uma acentuada diminuição imediata da dor lombar e da dor irradiada para a coxa esquerda após o primeiro ajuste da quiropraxia. Entretanto, ainda apresentava fraqueza na perna esquerda assim como uma diferença no tônus muscular e no tamanho da coxa esquerda. O mesmo tratamento quiropráxico foi aplicado por 6 semanas e complementado por 12 semanas de estimulação elétrica dos músculos atrofiados e uma bateria de exercícios para melhora da função motora. Após as 12 semanas, houve completa resolução da atrofia muscular, não havia sensação de instabilidade no joelho, e o reflexo patelar foi normalizado.

Outro estudo ${ }^{14}$ examinou os efeitos e custos a longo prazo da combinação de tratamento manipulativo, exercícios estabilizadores e consulta com médico (grupo combinado) comparado com a consulta médica apenas (grupo consulta), em 204 trabalhadores de 24 a 46 anos de idade com dor lombar crônica (com ou sem dor ciática). Os pacientes do grupo combinado realizaram uma avaliação, tratamento e sessões de exercícios uma vez por semana durante 4 semanas. A terapia incluía manipulação usando técnica de músculo-energia e exercícios de controle motor visando corrigir o ritmo lombopélvico. Ambos os grupos, combinação e consulta, foram examinados clinicamente, com todos os achados clínicos e radiográficos explicados aos pacientes. Cada paciente recebeu um livreto educacional e instruções individuais a respeito da postura, além de três ou quatro exercícios para mobilidade vertebral, alongamento muscular e/ou estabilidade dos músculos do tronco, de acordo com os achados clínicos, a fim de incentivá-los a uma postura ativa. Essas informações foram reforçadas por 5 meses. Após 2 anos, a maior eficácia na redução da dor e na satisfação dos pacientes em relação ao tratamento foi verificada a favor do grupo combinado. A qualidade de vida melhorou igualmente em ambos os grupos, porém permanece abaixo do escore da população saudável. As visitas anuais aos médicos e fisioterapeutas diminuíram nos dois grupos. Entretanto, o grupo consulta apresentou uma economia maior nos gastos com saúde assim como no número de faltas anuais do trabalho por motivo de doença, melhorando a relação custo-benefício. 
Para Earl2, o tratamento da espondilolistese deve ser baseado na idade do paciente, no tempo de lesão, na progressão do deslizamento e no nível de atividade do paciente. $\mathrm{O}$ autor citou como exemplos de reabilitação conservadora da espondilolistese fortalecimento abdominal, treino da postura e de padrão de movimento, alongamento dos isquiotibiais e psoas e treino da estabilidade pélvica. Além disso, um colete lombossacro leve pode ser usado em conjunto com a reabilitação. Também é importante limitar as atividades de alto risco e avaliar posteriormente, para monitorar a progressão da listese.

Lonstein ${ }^{3}$ apontou condutas semeIhantes no tratamento da espondilolistese em crianças, sendo a combinação de colete e exercícios muito documentada. Ele verificou que, apesar das séries relatadas normalmente serem pequenas e do curto período de acompanhamento, os resultados foram geralmente bons em dois terços ou mais das crianças. $\mathrm{O}$ período de uso do colete nos estudos por ele revisados variou de 2 meses no mínimo até 25 meses em média. Após a resolução da dor, a criança podia voltar a atividades esportivas e realizar programas de exercícios terapêuticos, que incluíam alongamento dos isquiotibiais e da fáscia lombodorsal, inclinações pélvicas, fortalecimento abdominal e exercícios de flexão de Williams. O sucesso do tratamento também dependia do grau de deslizamento, com bons resultados no alívio da dor apenas até grau II.

Standaert e Herring ${ }^{12}$ encontraram variação maior na aplicabilidade, tipo e tempo de uso de coletes em atletas adolescentes com espondilólise. O tempo de uso variou de poucas semanas até um ano para esse tipo de paciente. O tipo permutou de coletes rígidos a corseletes leves. Contudo, estudos biomecânicos dos efeitos do colete não apoiaram seu uso particular na espondilólise, porque muitos dos pacientes que foram colocados em um colete lombossacro rígido na realidade experimentaram uma redução no movimento intervertebral da articulação lombossacra. O principal efeito do colete parece estar na restrição do movimento global do corpo. Dessa forma, o colete agiria mais como um meio de restringir a atividade do que estabilizando as fraturas desses pacientes. A estrutura básica de tratamento da espondilólise em atletas adolescentes sugerida pelos autores incluía, primeiramente, um descanso relativo, uma vez que a patologia resulta do estresse excessivo no osso. O tempo de restrição das atividades depende do quadro clínico e das imagens diagnósticas, sendo aconselhado manter-se até a resolução da dor lombar. Um colete rígido pode ser recomendado após duas semanas de descanso se os sintomas não estiverem melhorando. A reabilitação dos atletas começa após o descanso adequado, com foco inicial no treinamento cardiovascular de baixo impacto, treino precoce da estabilização e avaliação de cadeia cinética mais ampla. Gradualmente, progride-se para um trabalho de estabilidade mais avançado, trabalho cardiovascular mais agressivo e retreinamento esporte-específico, que enfatiza o desempenho funcional e a correção biomecânica. Esse tipo de treinamento reduz a quantidade e intensidade de atividades específicas que possam contribuir para o desenvolvimento de lesão na pars interarticularis. Os atletas são liberados para voltar ao esporte quando completam a amplitude de movimento sem dor, alcançam o condicionamento apropriado, consciência corporal e demonstram habilidades esporte-específicas em ambiente controlado, sem dor. Geralmente, a reabilitação requer de 2 a 4 meses para se completar, resultando num retorno ao esporte aproximadamente 5 a 7 meses após o diagnóstico.

\section{DISCUSSÃO}

Até $80 \%$ da população pode ter dor lombar em algum momento1, porém a prevalência de cirurgia da coluna lombar durante a vida é de somente $1 \%$ a $3 \% 1$. Além disso, estudos da história natural da patologia notaram que a maioria dos pacientes com espondilolistese não pioram com o tempo e muito raramente ocorre uma rápida deterioração ${ }^{3}$. Conseqüentemente, o tratamento não-cirúrgico é o principal tratamento para dor lombar e dever ser a ação inicial na maioria dos casos de espondilolistese. Não há, entretanto, nenhum estudo prospectivo randomizado de experimentações clínicas que estabeleça um protocolo ideal de tratamento conservador 5 .
Dos sete artigos selecionados, três estudos abordaram tratamento com terapia manual, quiropraxia e/ou osteopatia. Destes, todos apresentaram resultados a favor da terapia manual ou da combinação dela com outras terapias na resolução dos sintomas e na melhora funcional do paciente com espondilólise/listese.

Foi demonstrado que a combinação de manipulação espinhal da quiropraxia, aplicação de modalidades terapêuticas para alívio dos sintomas e reabilitação física podem ser eficazes na gerência das espondilolisteses difíceis associadas com lesões progressivas do disco em múltiplos níveis 16 .

Dos numerosos estudos com manipulação espinhal, somente poucos são randomizados e controlados com acompanhamento superior a um ano17-20; encontrou-se um predomínio da abordagem fisioterapêutica convencional e uma escassez de estudos publicados sobre o uso da terapia manual para o tratamento da espondilólise e espondilolistese.

Estudos têm identificado a fisioterapia como o método mais comum de tratamento não-cirúrgico da espondilólise/ listese, com bons resultados 21,22. Entretanto, poucos estudos prospectivos foram realizados em que a eficácia da fisioterapia fosse examinada nesse tópico ${ }^{5}$.

Em ambas as vertentes de tratamento, fisioterapia convencional e outras terapias manuais, estudos controlados randomizados são necessários e deveriam estender-se à avaliação da eficácia das modalidades de tratamento. Também, para efetivamente estudar essas condições, a resposta ao tratamento deveria ser avaliada com indivíduos de idades diferentes e em estágios diferentes da condição, o que torna imprescindível a realização de estudos multicêntricos. Além disso, mais pesquisas também são necessárias para a identificação da etiologia da espondilólise e espondilolistese e para determinação da relação entre instabilidade e sintomatologia apresentada. Isso auxiliaria muito na prevenção, detecção precoce e escolha do tratamento apropriado.

Tempo de exposição aumentado e participação em esportes ou atividades intensas, entre crianças e adolescentes, foram correlacionados com aumento da 
dor lombar relatada23. Na sociedade atual, é crescente o número de praticantes de esportes radicais, ciclismo, artes marciais e outros que requerem hiperextensão ou hiperflexão da coluna lombar. Diante dessa tendência, torna-se mais provável um aumento no número de indivíduos com espondilólise e espondilolistese, o que implica maior necessidade de estudos envolvendo especificamente a população atlética jovem.

Os achados desta revisão são específicos para dor lombar devido à espondilólise e espondilolistese e para o campo da fisioterapia e terapias manuais. Como a busca focalizou estudos publicados nas línguas portuguesa e inglesa, os estudos encontrados podem não representar toda a pesquisa disponível na área, mas constitui uma amostra representativa.

Outra limitação ocorreu devido à pequena quantidade de pesquisas com critérios rigorosos de relevância científica. Como resultado, o fator de seleção ateve-se aos critérios metodológicos relacionados com a significância clínica (sujeitos, descrição dos tratamentos e a avaliação dos desfechos escolhidos). Assim, chama a atenção o fato de os autores de estudos clínicos precisarem publicar sua metodologia de estudo e resultados com detalhes suficientes que permitam análises de rigor científico e replicabilidade.

\section{CONCLUSÃO}

Tanto o tratamento conservador por fisioterapia convencional como terapias manuais apresentaram efeitos benéficos na redução da lombalgia e na melhora funcional do paciente. As terapias manuais envolveram manipulação da coluna cervical, torácica, lombossacra e articulação sacroilíaca, músculo-energia e alongamento dos músculos afetados.

Houve consenso entre os artigos que exercícios de estabilização lombopélvica, fortalecimento dos músculos posturais axiais e alongamento dos isquiotibiais, psoas e fáscia lombodor- sal são importantes no tratamento da espondilólise/listese. Procedimentos para alívio dos sintomas, como calor, massagem, ultra-som e TENS, também foram eficazes quando em combinação com um programa de reabilitação.

Permanece controversa a indicação do colete, assim como a prescrição de exercícios apenas em flexão ou extensão. O paciente deve ser avaliado individualmente em seu quadro clínico e radiográfico para determinação do plano de tratamento.

Dentre as opções conservadoras de tratamento encontradas, nenhuma se mostrou conclusivamente superior às outras e todas têm um papel no tratamento dos pacientes sintomáticos com espondilólise/listese.

Assim, como os resultados desta revisão da literatura dos últimos dez anos sobre o tratamento conservador da espondilólise e espondilolistese são limitados, o campo permanece aberto e amplo para futuras pesquisas. 


\section{REFERÊNCIAS}

1 Resnick D. Diagnosis of bone and joint disorders. 3rd ed. Philadelphia: WB Saunders; 1995.

2 Earl JE. Mechanical etiology, recognition, and treatment of spondylolisthesis. Phys Ther Sport. 2002;3(2):79-87.

3 Lonstein JE. Spondylolisthesis in children: cause, natural history, and management. Spine. 1999;24(24):2640-8.

4 Dickson RA. Spine: spondylolisthesis. Curr Orthop. 1998;12(4):273-82.

5 McNeely ML, Torrance G, Magee DJ. A systematic review of physiotherapy for spondylolysis and spondylolisthesis. Man Ther. 2003;8(2):80-91.

6 Johnson RJ. Low back pain in sports. Phys Sportsmed. 1993;21(4):53-9.

7 Stinson JT. Spondylolysis and spondylolisthesis in the athlete. Clin Sports Med. 1993;12(3):517-28.

8 King HA. Back pain in children. Orthop Clin North Am. 1999;30(3):467-74.

9 Wiltse LL, Newman PH, Macnab I. Classification of spondylolysis and spondylolisthesis. Clin Orthop Relat Res. 1976;117:23-9.

10 Vialle R, Benoist M. High-grade lumbosacral spondylolisthesis in children and adolescents: pathogenesis, morphological analysis, and therapeutic strategy. Joint Bone Spine. 2007;74(5)414-7.

11 Magee DJ. Avaliação musculoesquelética. 4a ed. Barueri: Manole; 2005.

12 Standaert C), Herring SA. Expert opinion and controversies in sports and musculoskeletal medicine: the diagnosis and treatment of spondylolysis in adolescent athletes. Arch Phys Med Rehabil. 2007;88(4):537-40.

13 Fritz JM, Erhard RE, Hagen BF. Segmental instability of the lumbar spine. Phys Ther. 1998;78(8):889-96.

14 Niemistö L, Rissanen P, Sarna S, Lahtinen-Suopanki T, Lindgren K-A, Hurri H. Cost-effectiveness of combined manipulation, stabilizing exercises, and physician consultation compared to physician consultation alone for chronic low-back pain: a prospective randomized trial with 2-year follow-up. Spine. 2005;30(10):1109-15.
15 Sinaki M, Lutness MP, Ilstrup DM, Chu CP, Gramse RR. Lumbar spondylolisthesis: retrospective comparison and three-year follow-up of two conservative treatment programs. Arch Phys Med Rehabil. 1989;70(8):594-8.

16 Excoffon SG, Wallace $\mathrm{H}$. Chiropractic and rehabilitative management of a patient with progressive lumbar disk injury, spondylolisthesis, and spondyloptosis. J Manipulative Physiol Ther. 2006;29(1):66-71.

17 Cherkin DC, Deyo RA, Battie M, Street J, Barlow W. A comparison of physical therapy, chiropractic manipulation, and provision of an educational booklet for the treatment of patients with low-back pain. $\mathrm{N}$ Engl J Med. 1998; 339(15):1021-9.

18 Skargren El, Carlsson PG, Oberg BE. One-year follow-up comparison of the cost and effectiveness of chiropractic and physiotherapy as primary management for back pain: subgroup analysis, recurrence, and additional health care utilization. Spine. 1998;23(17):1875-84.

19 Burton AK, Tillotson KM, Cleary J. Single-blind randomised controlled trial of chemonucleolysis and manipulation in the treatment of symptomatic lumbar disc herniation. Eur Spine J. 2000;9(3):202-7.

20 Hemmila HM, Keinänen-Kiukaanniemi SM, Levoska S, Puska P. Long-term effectiveness of bone-setting, light exercise therapy, and physiotherapy for prolonged back pain: a randomized controlled trial. J Manipulative Physiol Ther. 2002;25(2):99-104.

21 Fellander-Tsai L, Micheli LJ. Treatment of spondylolysis with external electrical stimulation and bracing in adolescent athletes: a report of two cases. Clin J Sport Med. 1998;8(3):232-4.

22 Szapalski M, Gunzberg R, Pope M. Lumbar segmental instability. Philadelphia: Lippincott, Williams \& Wilkins; 1999.

23 Motley G, Nyland J, Jacobs J, Caborn DNM. The pars interarticularis stress reaction, spondylolysis and spondylolisthesis progression. J Athl Train. 1998;33(4):351-8. 